\title{
A Randomized Phase II Study of Leucovorin/5-Fluorouracil with or without Oxaliplatin (LV5FU2 vs. FOLFOX) for Curatively-Resected, Node-Positive Esophageal Squamous Cell Carcinoma
}

\author{
Sung Hee Lim, MD', \\ Young Mog Shim, MD, PhD \\ Se Hoon Park, MD, PhD² \\ Hong Kwan Kim, MD, PhD 3 \\ Young Soo Choi, MD, $\mathrm{PhD}^{3}$ \\ Myung-Ju Ahn, MD, PhD2 \\ Keunchil Park, MD, PhD² \\ Jae III Zo, MD, PhD ${ }^{3}$ \\ Jong-Mu Sun, $\mathrm{MD}, \mathrm{PhD}{ }^{2}$
}

${ }^{1}$ Division of Hematology-Oncology,

Department of Internal Medicine,

Dongtan Sacred Heart Hospital, Hallym

University of College of Medicine, Hwaseong,

${ }^{2}$ Division of Hematology-Oncology,

Department of Medicine, ${ }^{3}$ Department of

Thoracic and Cardiovascular Surgery,

Samsung Medical Center, Sungkyunkwan

University School of Medicine, Seoul, Korea

\section{Correspondence: Jong-Mu Sun, MD, PhD \\ Division of Hematology-Oncology, \\ Department of Medicine, Samsung Medical \\ Center, Sungkyunkwan University School \\ of Medicine, 81 Irwon-ro, Gangnam-gu, \\ Seoul 06351, Korea \\ Tel: 82-2-3410-1795 \\ Fax: 82-2-3410-1754 \\ E-mail: tntntn3@gmail.com}

Received August 30, 2016

Accepted October 26, 2016

Published Online November 9, 2016

*Sung Hee Lim and Young Mog Shim

contributed equally to this work.

\begin{abstract}
Purpose
The optimal perioperative treatment for resectable esophageal squamous cell carcinoma (ESCC) remains controversial. We evaluated the efficacy and safety of leucovorin and 5-fluorouracil (LV5FU2) and LV5FU2 plus oxaliplatin (FOLFOX) combination chemotherapies administered adjuvantly for curatively-resected, node-positive ESCC.
\end{abstract}

\section{Materials and Methods}

Patients with pathologically node-positive esophageal cancer after curative RO resection were enrolled and randomly assigned to receive LV5FU2 or FOLFOX biweekly for up to eight cycles. The primary endpoint was disease-free survival (DFS).

\section{Results}

Between 2011 and 2015, 62 patients were randomized into the two treatment groups (32 in the LV5FU2 arm and 30 in the FOLFOX arm). The median age was 60 years and both groups had similar pathologic characteristics in tumor, nodal status, and location. Treatment completion rates were similarly high in both groups. The DFS rate at 12 months was $67 \%$ in the LV5FU2 group and 63\% in the FOLFOX group with a hazard ratio of 1.3 (95\% confidence interval [Cl], 0.66 to 2.62). After a median follow-up period of 27 months, the median DFS was 29.6 months ( $95 \% \mathrm{Cl}, 4.9$ to 54.2$)$ in the LV5FU2 arm and 16.8 months $(95 \% \mathrm{Cl}$, 7.5 to 26.1 ) in the FOLFOX arm $(p=0.428)$, respectively, while the median overall survival was not reached in either arm. Grade 3 or 4 neutropenia was more frequent in patients in the FOLFOX arm than the LV5FU2 arm (20.0\% vs. 3.1\%).

\section{Conclusion}

The addition of oxaliplatin (FOLFOX) did not lead to better efficacy compared to LV5FU2 chemotherapy in an adjuvant setting in node-positive ESCC patients.

\section{Introduction}

Esophageal cancer is the sixth most common cause of cancer death worldwide [1] and remains a significant unmet medical need in Korea [2] with an overall poor prognosis. Esophageal cancers are histologically classified as squamous cell carcinoma (SCC) or adenocarcinoma, and SCC, which has risk factors including smoking and alcohol abuse, is the
Key words

Esophageal neoplasms, Adjuvant chemotherapy, FOLFOX most common form of esophageal cancer in Asian countries [3]. Surgery is the primary course of treatment for resectable disease, and lymph node metastasis has been shown to be a strong independent predictor of poor survival [4]. Although surgery is the mainstay of curative treatment, surgery alone has limited efficacy at improving long-term survival in locally advanced esophageal cancer, especially node-positive disease. Therefore, more effective multimodal treatment methods, including neoadjuvant or adjuvant chemotherapy, 
radiotherapy and chemoradiotherapy (CRT) have been investigated for their potential to improve overall survival (OS).

Neoadjuvant chemotherapy or concurrent CRT followed by operation have been regarded as standard treatments for locally advanced esophageal cancer [5-8]. However, many patients have been found to have advanced, node-positive, esophageal cancer only after they received upfront surgery without neoadjuvant therapy because of the incomplete accuracy of preoperative tumor staging. For example, one report showed that 81 esophageal cancer patients were staged to have node-negative disease by positron emission tomography, chest computed tomography, and/or endoscopic ultrasonography and received upfront surgery without neoadjuvant therapy. However, 37 of these patients were found to have pathological node(s) in the surgical specimens [9].

For this population, adjuvant cisplatin and fluorouracil (FP) is recommended based on the improved disease-free survival (DFS) associated with adjuvant FP in patients with node-positive esophageal SCC [10]. However, most patients with esophageal cancer are elderly with poor nutritional status and become fragile after receiving major operations; therefore, adjuvant FP can be too toxic a regimen for this population [11]. Accordingly, it is necessary to identify an adjuvant chemotherapy regimen that is more tolerable but comparably efficient with FP. In this study, we prospectively compared the combination regimen of fluorouracil and leucovorin, and oxaliplatin (FOLFOX) with a non-platinum regimen of fluorouracil and leucovorin (LV5FU2) to evaluate whether FOLFOX can be an acceptable adjuvant regimen for locally advanced esophageal SCC.

\section{Materials and Methods}

\section{Patients and study design}

This study was a single-center, open-label, randomized phase II trial (NCT01467921) of patients aged 20 years or older with histologically confirmed SCC of the esophagus. All patients underwent curative esophagectomy and regional lymphadenectomy and had pathologically nodepositive disease. Patients who received prior neoadjuvant chemotherapy, radiotherapy or concurrent chemoradiation (CCRT) before registration were excluded. Eligible patients had an Eastern Cooperative Oncology Group (ECOG) performance status of 0 or 1 and adequate organ function. This study was approved by the Institutional Review Board of Samsung Medical Center. Written informed consent was acquired from each patient before enrollment. Patients with previous oxaliplatin use, severe co-morbid illnesses, and/or active infections were also excluded. All pathological staging of tumors for patients was based on the seventh edition of the American Joint Committee on Cancer (AJCC) tumornode-metastasis (TNM) classification guidelines.

\section{Study treatment}

Four to six weeks after surgery, eligible patients were randomized to receive (1:1) LV5FU2 or FOLFOX chemotherapy for up to eight cycles or until disease progression before completion of adjuvant treatment, unacceptable toxicity, or patient withdrawal. The LV5FU2 regimen consisted of 2-week cycles of $200 \mathrm{mg} / \mathrm{m}^{2}$ leucovorin plus and a bolus injection of 5-fluorouracil (5-FU; $400 \mathrm{mg} / \mathrm{m}^{2}$ ) intravenously on day 1 , followed by a 46 -hour continuous infusion of 5 -FU $\left(2,400 \mathrm{mg} / \mathrm{m}^{2}\right)$. In the FOLFOX arm, 2-week cycles of oxaliplatin $85 \mathrm{mg} / \mathrm{m}^{2}$ were given intravenously on day 1 before leucovorin and 5-FU and the dose of 5-FU/leucovorin was the same as LV5FU2 regimen. If patients showed disease progression during or after the study treatment, further treatment could be administered at the discretion of the investigators.

\section{Endpoints}

The primary endpoint of the study was DFS, which was defined as the time from the date of registration to the date of recurrence, death, or last contact. Secondary endpoints included OS, safety and quality of life assessment. OS was calculated from the time of randomization to the date of death. These endpoints were measured in all registered patients (i.e., the intention-to-treat population). Recurrence and survival data were evaluated every four cycles during the study treatment and every 3 months after completion of the study treatment. Safety evaluation included all patients who received at least one dose of study drug and adverse events (AEs) were assessed according to the National Cancer Institute criteria Common Terminology Criteria for Adverse Events (CTCAE) ver. 3 [12].

\section{Statistical analysis}

Assuming a 12-month DFS rate of $60 \%$ for the LV5FU2 arm, this study required a total of 56 patients (28 per arm) with $90 \%$ power to detect a $20 \%$ difference in 12 -month DFS using the two-sided log-rank test with an alpha level of 0.05 . A 20\% ineligible or non-assessable rate was assumed, resulting in an accrual goal of 68 patients (34 patients for each arm). For DFS and OS, survival functions were estimated using the Kaplan-Meier method and compared between 


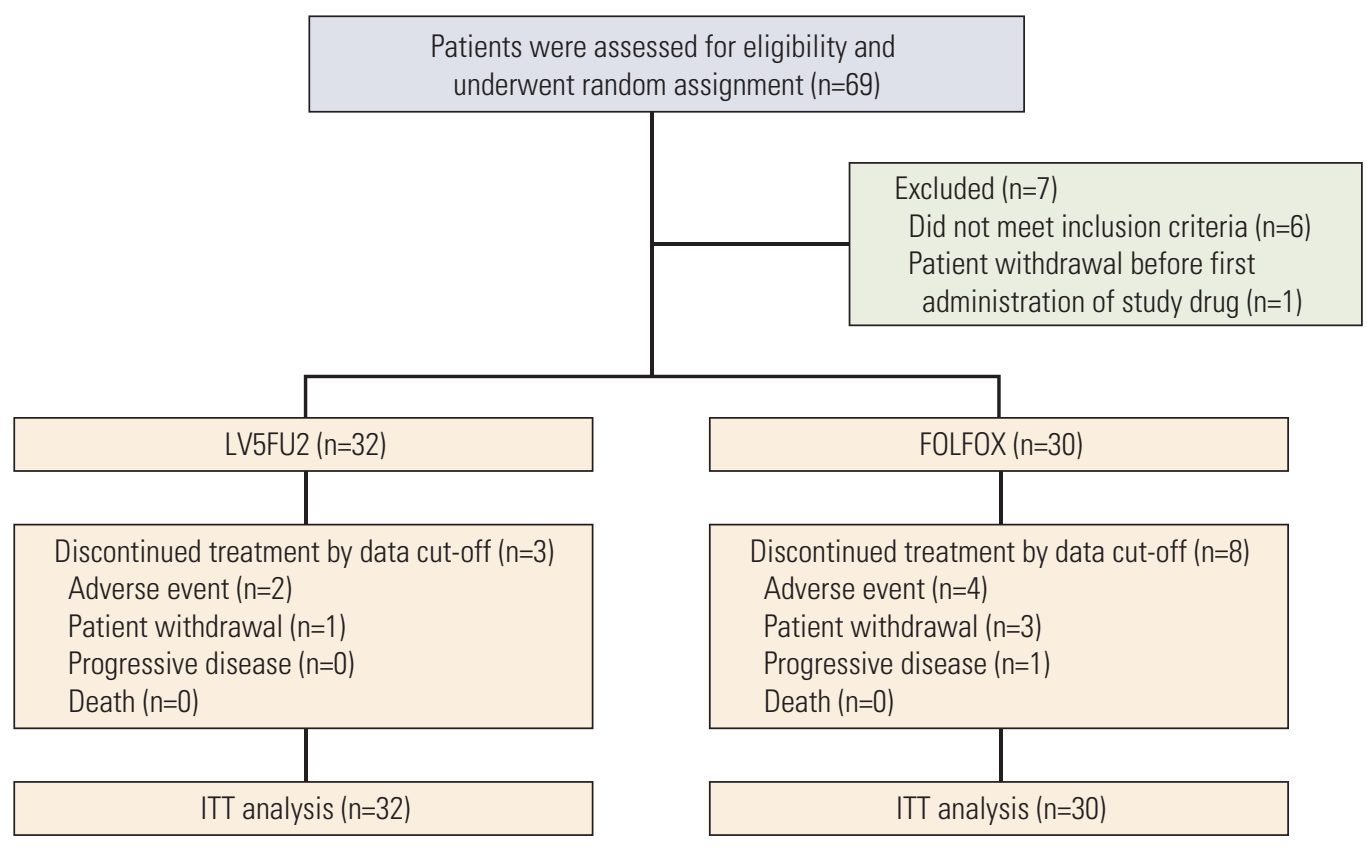

Fig. 1. CONSORT diagram of the study design and participants. LV5FU2, leucovorin and 5-fluorouracil; FOLFOX, fluorouracil, leucovorin, and oxaliplatin; ITT, intention-to-treat.

groups via the log-rank test. $\mathrm{p}<0.05$ were considered statistically significant. Cox regression analysis was used for univariate and multivariate analysis to identify significant prognostic factors for recurrence. All reported p-values are two-sided and were calculated using SPSS ver. 21 (IBM Corp., Armonk, NY).

\section{Results}

\section{Study patients}

Between January 2011 and March 2015, 62 patients were randomly assigned to receive either LV5FU2 $(n=32)$ or FOLFOX ( $n=30)$ chemotherapy (Fig. 1). Among a total of 69 patients enrolled, one patient was excluded due to patient withdrawal and six were removed owing to preoperative treatment. The clinicopathologic characteristics of all patients are listed in Table 1. All patients in both arms had SCC of the esophagus and pathologically confirmed regional lymph node metastasis. The median age of all patients was 60 years (range, 43 to 77 years). Most were male (97\%), and all patients had an ECOG performance status of 1 . Both groups had similar pathological characteristics with regard to tumor, nodal status, and location. Approximately $70 \%$ of patients had stage III disease, and the upper esophagus tumors represented $20 \%$ of patients in the LV5FU2 group and $33 \%$ of the FOLFOX group.

\section{Efficacy}

At the data cutoff point (February 2016), the median follow-up duration was 27.0 months (range, 4.5 to 59.1 months). The DFS rate at 12 months, which was the primary endpoint in this study, was $67 \%$ (95\% confidence interval [CI], 50.7 to 83.3) in the LV5FU2 group and 63\% (95\% CI, 45.7 to 80.3$)$ in the FOLFOX group with a hazard ratio (HR) of $1.3(95 \% \mathrm{CI}$, 0.66 to 2.62). Specifically, the median DFS was 29.6 months (95\% CI, 4.9 to 54.2 ) in the LV5FU2 arm and 16.8 months (95\% CI, 7.5 to 26.1) in the FOLFOX arm ( $\mathrm{p}=0.428$ ) (Fig. 2A), respectively, while the median OS was not reached in either arm ( $p=0.904$ ) (Fig. 2B). The estimated 3-year DFS and OS were $46 \%$ and $57 \%$ in the LV5FU2 group and $39 \%$ and $59 \%$ in the FOLFOX group, respectively.

In total, 15 patients in the LV5FU2 group and 18 in the FOLFOX group recurred or progressed during follow-up. There were no significant differences in the pattern of recurrence between groups, although both local and distant recurrence were observed in six patients in the FOLFOX arm (Table 2). Univariate analysis revealed significant risk factors for tumor recurrence including advanced nodal status (N3, $\mathrm{p}=0.020)$, tumor status $(\mathrm{T} 3, \mathrm{p}=0.005)$, and stage III $(\mathrm{p}=0.019)$ 
Table 1. Baseline patient characteristics

\begin{tabular}{|c|c|c|}
\hline Characteristic & LV5FU2 (n=32) & FOLFOX $(n=30)$ \\
\hline \multicolumn{3}{|l|}{ Age (yr) } \\
\hline Mean \pm SD & $61.9 \pm 6.11$ & $60.0 \pm 7.84$ \\
\hline Range & $46-73$ & 43-77 \\
\hline \multicolumn{3}{|l|}{ Sex } \\
\hline Male & $31(96.9)$ & $29(96.7)$ \\
\hline Female & $1(3.1)$ & $1(3.3)$ \\
\hline \multicolumn{3}{|l|}{ ECOG performance status } \\
\hline 1 & $32(100)$ & $30(100)$ \\
\hline \multicolumn{3}{|l|}{ Histology } \\
\hline Squamous cell carcinoma & $32(100)$ & $30(100)$ \\
\hline \multicolumn{3}{|l|}{ Differentiation } \\
\hline Well & 7 (21.9) & $3(10.0)$ \\
\hline Moderate & $22(68.8)$ & $20(66.7)$ \\
\hline Poorly & $2(6.2)$ & $6(20.0)$ \\
\hline Undifferentiated & $1(3.1)$ & $1(3.3)$ \\
\hline \multicolumn{3}{|l|}{ Pathologic tumor status } \\
\hline $\mathrm{T} 1$ & $11(34.4)$ & $11(36.7)$ \\
\hline $\mathrm{T} 1 \mathrm{a} / \mathrm{T} 1 \mathrm{~b}$ & $1 / 8$ & $0 / 8$ \\
\hline $\mathrm{T} 2$ & $6(18.8)$ & $2(6.7)$ \\
\hline T3 & $15(46.9)$ & $17(56.7)$ \\
\hline \multicolumn{3}{|l|}{ Pathologic nodal status } \\
\hline N1 & $14(43.8)$ & $17(56.7)$ \\
\hline N2 & $13(40.6)$ & $9(30.0)$ \\
\hline N3 & $5(15.6)$ & $4(13.3)$ \\
\hline \multicolumn{3}{|l|}{ Pathologic staging } \\
\hline IIB & $9(28.1)$ & $11(36.7)$ \\
\hline III & $23(71.9)$ & $19(63.3)$ \\
\hline IIIA/IIIB/IIIC & $10 / 8 / 5$ & $8 / 7 / 4$ \\
\hline \multicolumn{3}{|l|}{ Location } \\
\hline Upper & $6(18.8)$ & $10(33.3)$ \\
\hline Middle & $11(34.4)$ & $8(26.7)$ \\
\hline Lower & $15(46.9)$ & $12(26.7)$ \\
\hline
\end{tabular}

Values are presented as number (\%). LV5FU2, leucovorin and 5-fluorouracil; FOLFOX, fluorouracil, leucovorin, and oxaliplatin; ECOG, Eastern Cooperative Oncology Group.

(Table 3). Middle esophageal cancers were associated with better DFS than upper esophageal cancers $(p=0.032)$.

\section{Toxicity}

All enrolled patients were monitored for adverse effects. The overall incidence of grade $\geq 3$ AEs was $9.4 \%$ (3 of 32 patients) in the LV5FU2 group and $23.3 \%$ (7 of 30 patients) in the FOLFOX group (Table 4). The most common AEs experienced in both treatment arms were neutropenia, thrombocytopenia, nausea, diarrhea, and anorexia. Grade 3 or higher neutropenia was more frequent in patients in the FOLFOX arm compared with the LV5FU2 arm (20.0\% vs.
$3.1 \%$ ), but no patients exhibited febrile neutropenia. The addition of oxaliplatin resulted in more frequent fatigue and peripheral neuropathy. Grade 3 aspiration events occurred in one case in each arm but were not related to aspiration pneumonia.

\section{Treatment and drug delivery}

The median number of chemotherapy cycles administered was eight (range, 4 to 8 ) in the LV5FU2 group and eight (range, 2 to 8 ) in the FOLFOX group. Treatment completion rates were similarly high in both groups (84\% in the LV5FU2 arm and $70 \%$ in the FOLFOX arm, $\mathrm{p}=0.230$ ). The delivered 
A
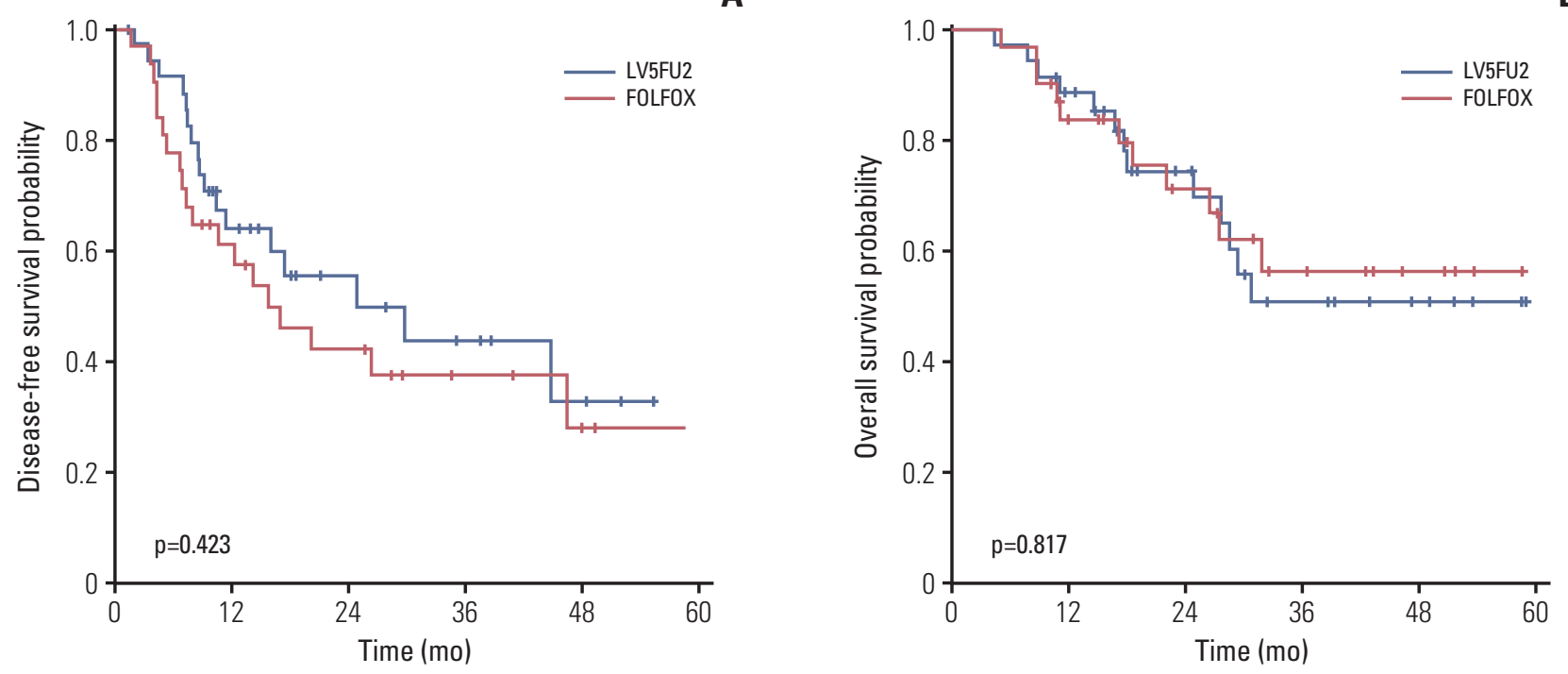

Fig. 2. (A) Kaplan-Meier curves of disease-free survival for patients who received FOLFOX or LV5FU2 (median diseasefree survival, 16.8 months in the FOLFOX arm vs. 29.6 months in the LV5FU2 arm). (B) Kaplan-Meier curves of overall survival of all patients according to treatment arm (median overall survival was not reached in either arm). FOLFOX, fluorouracil, leucovorin, and oxaliplatin; LV5FU2, leucovorin and 5-fluorouracil.

Table 2. Pattern of failure

\begin{tabular}{|c|c|c|}
\hline Site of recurrence & $\begin{array}{c}\text { LV5FU2 } \\
(\mathrm{n}=15)\end{array}$ & $\begin{array}{c}\text { FOLFOX } \\
(\mathrm{n}=18)\end{array}$ \\
\hline \multicolumn{3}{|l|}{ Loco-regional } \\
\hline Cervical lymph node & 1 & 1 \\
\hline Mediastinal lymph node & 2 & - \\
\hline Abdominal lymph node & 1 & 1 \\
\hline Lymph node, others & 7 & 3 \\
\hline \multicolumn{3}{|l|}{ Distant } \\
\hline Lung & 1 & 5 \\
\hline Peritoneum & 1 & 1 \\
\hline Liver & 2 & 3 \\
\hline Bone & 1 & 1 \\
\hline Other & 1 & 2 \\
\hline Both local and distant & - & 6 \\
\hline
\end{tabular}

LV5FU2, leucovorin and 5-fluorouracil; FOLFOX, fluorouracil, leucovorin, and oxaliplatin.

relative dose intensities for oxaliplatin, fluorouracil and leukovorin were similarly high in both treatment groups (S1 Table). Reductions in the dose of the randomly assigned treatment were performed in $21.9 \%$ of all patients in the LV5FU2 group and $60.0 \%$ in the FOLFOX group, whereas
$3.1 \%$ of all patients in the LV5FU2 group and $26.7 \%$ in the FOLFOX group experienced delays in the assigned treatment.

\section{Discussion}

Prognosis is poor in most patients treated with curative resection of esophageal cancer who present with local and / or distant recurrence during follow-up. One Japanese study showed that two cycles of FP chemotherapy yielded a much greater survival benefit when administered before surgery than after in locally advanced esophageal SCC [13]. In addition, a recent meta-analysis showed that neoadjuvant CRT (HR, $0.8 ; \mathrm{p}=0.002)$ and neoadjuvant chemotherapy (HR, $0.89 ; \mathrm{p}=0.051$ ) were associated with increased OS in locally advanced esophageal SCC [14]. Thus, neoadjuvant chemotherapy or CRT has attracted more interest clinically than adjuvant therapy. However, adjuvant FP chemotherapy has also been suggested to improve DFS, especially in patients with pathologically node-positive esophageal SCC, although this benefit did not extend to OS [10]. There are also many clinical situations in which chemotherapy or CRT cannot be administered before surgery and adjuvant therapy is 
Table 3. Univariate analysis of the DFS and OS

\begin{tabular}{|c|c|c|c|c|c|c|}
\hline \multirow{2}{*}{ Predictive factor } & \multicolumn{3}{|c|}{ DFS } & \multicolumn{3}{|c|}{ OS } \\
\hline & HR & $95 \% \mathrm{CI}$ & p-value & HR & $95 \% \mathrm{CI}$ & p-value \\
\hline Age $(\geq 60$ yr vs. $<60$ yr $)$ & 0.88 & $0.44-1.76$ & 0.712 & 1.27 & $0.51-3.19$ & 0.609 \\
\hline Group (FOLFOX vs. LV5FU2) & 1.32 & 0.662 .62 & 0.430 & 1.06 & $0.44-2.54$ & 0.904 \\
\hline \multicolumn{7}{|l|}{ Location } \\
\hline Middle vs. upper & 0.35 & 0.140 .92 & 0.032 & 0.16 & $0.03-0.77$ & 0.022 \\
\hline Lower vs. upper & 0.58 & 0.261 .30 & 0.187 & 0.57 & $0.22-1.47$ & 0.246 \\
\hline \multicolumn{7}{|l|}{ Differentiation } \\
\hline Moderate vs. well & 0.57 & 0.241 .35 & 0.200 & 0.56 & $0.20-1.55$ & 0.263 \\
\hline Poorly vs. well & 0.54 & $0.16-1.85$ & 0.326 & 0.21 & $0.03-1.83$ & 0.159 \\
\hline \multicolumn{7}{|l|}{ T stage } \\
\hline T2 vs. T1 & 0.86 & $0.18-4.12$ & 0.856 & 0.76 & $0.09-6.59$ & 0.807 \\
\hline T3 vs. T1 & 3.21 & $1.42-7.25$ & 0.005 & 2.64 & $0.95-7.38$ & 0.064 \\
\hline \multicolumn{7}{|l|}{ N stage } \\
\hline N2 vs. N1 & 1.55 & $0.71-3.37$ & 0.274 & 1.17 & $0.44-3.08$ & 0.755 \\
\hline N3 vs. N1 & 3.03 & $1.19-7.74$ & 0.020 & 1.41 & $0.39-5.16$ & 0.602 \\
\hline Stage (III vs. II) & 2.91 & $1.19-7.14$ & 0.019 & 1.32 & $0.51-3.44$ & 0.574 \\
\hline
\end{tabular}

DFS, disease-free survival; OS, overall survival; HR, hazard ratio; CI, confidence interval; FOLFOX, fluorouracil, leucovorin, and oxaliplatin; LV5FU2, leucovorin and 5-fluorouracil.

Table 4. Toxicity profile

\begin{tabular}{|c|c|c|c|c|}
\hline \multirow{2}{*}{ Toxicity } & \multicolumn{2}{|c|}{ LV5FU2 (n=32) } & \multicolumn{2}{|c|}{ FOLFOX $(n=30)$} \\
\hline & Any grade & G3-4 & Any grade & G3-4 \\
\hline \multicolumn{5}{|l|}{ Hematologic } \\
\hline Neutropenia & $10(31.2)$ & $1(3.1)$ & $18(60.0)$ & $6(20.0)$ \\
\hline Febrile neutropenia & - & - & - & - \\
\hline Anemia & $9(28.1)$ & - & $8(26.7)$ & - \\
\hline Leukopenia & $2(6.2)$ & - & $5(16.7)$ & - \\
\hline Thrombocytopenia & $7(21.9)$ & - & $20(66.6)$ & $1(3.3)$ \\
\hline \multicolumn{5}{|l|}{ Non-hematologic } \\
\hline Nausea & $14(43.8)$ & $1(3.1)$ & $18(60.0)$ & - \\
\hline Vomiting & 5 (15.6) & $1(3.1)$ & $5(16.7)$ & - \\
\hline Diarrhea & $12(37.5)$ & - & $10(33.3)$ & - \\
\hline Constipation & $2(6.2)$ & - & $2(6.7)$ & - \\
\hline Stomatitis & $2(6.2)$ & - & $3(10.0)$ & - \\
\hline Mucositis & $6(18.8)$ & - & $5(16.7)$ & - \\
\hline Peripheral neuropathy & $4(12.5)$ & - & $19(63.3)$ & - \\
\hline Alopecia & $1(3.1)$ & - & $2(6.7)$ & - \\
\hline Anorexia & $12(37.5)$ & - & $17(56.7)$ & - \\
\hline Pruritus & - & - & - & - \\
\hline Hand-foot syndrome & $1(3.1)$ & - & $1(3.3)$ & - \\
\hline Fatigue & $4(12.5)$ & - & $13(43.3)$ & - \\
\hline Insomnia & $4(12.5)$ & - & 4 (13.3) & - \\
\hline Hyperpigmentation & $1(3.1)$ & - & - & - \\
\hline Skin rash & $2(6.2)$ & - & $1(3.3)$ & - \\
\hline
\end{tabular}

Values are presented as number (\%). LV5FU2, leucovorin and 5-fluorouracil; FOLFOX, fluorouracil, leucovorin, and oxaliplatin. 
required after curative resection. Therefore, exploring which regimen is appropriate for adjuvant chemotherapy in patients undergoing curative resection for node-positive esophageal SCC could provide important clinical information.

The optimal chemotherapeutic agents and adequate number of cycles of adjuvant chemotherapy for node-positive esophageal SCC have not been established thus far, and the most commonly used FP regimen is associated with significant toxicity $[11,15]$. Given that many esophageal cancer patients are elderly and therefore sensitive to toxic chemotherapy after operation, we investigated the clinical role of an adjuvant FOLFOX regimen for esophageal SCC and compared it with a potentially less toxic regimen, 5-FU/leucovorin. In esophageal cancer, FOLFOX chemotherapy was introduced for use in patients with advanced or metastatic disease [16]. FOLFOX chemotherapy in the definitive CCRT was also recently shown to be more convenient and less toxic than a 5-FU plus cisplatin regimen [15].

However, in the present study, adjuvant FOLFOX chemotherapy did not improve DFS or OS relative to 5-FU/ leucovorin chemotherapy but was instead related to a slightly increased incidence of neutropenia and dose reductions or delays. A possible explanation for this negative result could be that over $50 \%$ of the enrolled patients had advanced nodal disease (N2, N3); thus, adjuvant treatment with chemotherapy alone may be insufficient to control locoregional recurrence (66.7\% for local recurrence, 33.3\% for distant recurrence). Hsu et al. [17] suggested that postoperative radiotherapy could improve OS for patients with advanced nodal disease. Adjuvant 5-FU/leucovorin was also better at preventing disease recurrence than we expected, with 3-year DFS and OS rates of $46 \%$ and $57 \%$, respectively, which were similar to the results of a previous trial using adjuvant FP $[13,18]$. Furthermore, many patients in the FOLFOX group did not complete the planned eight cycles of chemotherapy, and eight cycles of FOLFOX adjuvant chemotherapy might be insufficient to demonstrate the efficacy.
It should be noted that our study had some limitations. The LV5FU2 regimen used for the control arm is not a standard treatment. Although there is no consensus on optimal adjuvant chemotherapy for resected esophageal SCC, cisplatinbased combinations remain the most commonly used regimens. Recently, newer drugs such as taxane (paclitaxel, docetaxel) and platinum analogues (carboplatin) have been investigated for esophageal cancer in various settings. Neoadjuvant CCRT with paclitaxel plus carboplatin followed by surgery led to marked increases in OS in esophageal SCC [7].

\section{Conclusion}

In summary, the results of the present study suggest that postoperative chemotherapy with a FOLFOX regimen does not result in improved DFS compared with a LV5FU2 regimen for patients who undergo curative resection of pathologically node-positive esophageal SCC. Further prospective trials on adjuvant chemotherapy in a selected population undergoing esophageal cancer resection are needed.

\section{Electronic Supplementary Material}

Supplementary materials are available at Cancer Research and Treatment website (http:// www.e-crt.org).

\section{Conflicts of Interest}

Conflict of interest relevant to this article was not reported.

\section{References}

1. Jemal A, Bray F, Center MM, Ferlay J, Ward E, Forman D. Global cancer statistics. CA Cancer J Clin. 2011;61:69-90.

2. Oh CM, Won YJ, Jung KW, Kong HJ, Cho H, Lee JK, et al. Cancer statistics in Korea: incidence, mortality, survival, and prevalence in 2013. Cancer Res Treat. 2016;48:436-50.

3. Layke JC, Lopez PP. Esophageal cancer: a review and update. Am Fam Physician. 2006;73:2187-94.

4. Steup WH, De Leyn P, Deneffe G, Van Raemdonck D, Coose- mans W, Lerut T. Tumors of the esophagogastric junction: long-term survival in relation to the pattern of lymph node metastasis and a critical analysis of the accuracy or inaccuracy of pTNM classification. J Thorac Cardiovasc Surg. 1996;111: 85-94.

5. Sjoquist KM, Burmeister BH, Smithers BM, Zalcberg JR, Simes RJ, Barbour A, et al. Survival after neoadjuvant chemotherapy or chemoradiotherapy for resectable oesophageal carcinoma: 
an updated meta-analysis. Lancet Oncol. 2011;12:681-92.

6. van Hagen P, Hulshof MC, van Lanschot JJ, Steyerberg EW, van Berge Henegouwen MI, Wijnhoven BP, et al. Preoperative chemoradiotherapy for esophageal or junctional cancer. $\mathrm{N}$ Engl J Med. 2012;366:2074-84.

7. Shapiro J, van Lanschot JJ, Hulshof MC, van Hagen P, van Berge Henegouwen MI, Wijnhoven BP, et al. Neoadjuvant chemoradiotherapy plus surgery versus surgery alone for oesophageal or junctional cancer (CROSS): long-term results of a randomised controlled trial. Lancet Oncol. 2015;16: 1090-8.

8. Allum WH, Stenning SP, Bancewicz J, Clark PI, Langley RE. Long-term results of a randomized trial of surgery with or without preoperative chemotherapy in esophageal cancer. J Clin Oncol. 2009;27:5062-7.

9. Yano M, Motoori M, Tanaka K, Kishi K, Fujiwara Y, Shingai $\mathrm{T}$, et al. Preoperative staging of clinically node-negative esophageal cancer by the combination of 18F-fluorodeoxyglucose positron emission tomography and computed tomography (FDG-PET/CT). Esophagus. 2012;9:210-6.

10. Ando N, lizuka T, Ide H, Ishida K, Shinoda M, Nishimaki T, et al. Surgery plus chemotherapy compared with surgery alone for localized squamous cell carcinoma of the thoracic esophagus: a Japan Clinical Oncology Group Study: JCOG9204. J Clin Oncol. 2003;21:4592-6.

11. Bleiberg H, Conroy T, Paillot B, Lacave AJ, Blijham G, Jacob $\mathrm{JH}$, et al. Randomised phase II study of cisplatin and 5-fluorouracil (5-FU) versus cisplatin alone in advanced squamous cell oesophageal cancer. Eur J Cancer. 1997;33:1216-20.

12. Shiozaki A, Yamagishi H, Itoi H, Fujiwara H, Kikuchi S, Okamoto $\mathrm{K}$, et al. Long-term administration of low-dose cis- platin plus 5-fluorouracil prolongs the postoperative survival of patients with esophageal cancer. Oncol Rep. 2005;13:667-72.

13. Ando N, Kato H, Igaki H, Shinoda M, Ozawa S, Shimizu H, et al. A randomized trial comparing postoperative adjuvant chemotherapy with cisplatin and 5-fluorouracil versus preoperative chemotherapy for localized advanced squamous cell carcinoma of the thoracic esophagus (JCOG9907). Ann Surg Oncol. 2012;19:68-74.

14. Pasquali S, Yim G, Vohra RS, Mocellin S, Nyanhongo D, Marriott $\mathrm{P}$, et al. Survival after neoadjuvant and adjuvant treatments compared to surgery alone for resectable esophageal carcinoma: a network meta-analysis. Ann Surg. 2017;265: 481-91.

15. Conroy T, Galais MP, Raoul JL, Bouche O, Gourgou-Bourgade $S$, Douillard JY, et al. Definitive chemoradiotherapy with FOLFOX versus fluorouracil and cisplatin in patients with oesophageal cancer (PRODIGE5/ACCORD17): final results of a randomised, phase 2/3 trial. Lancet Oncol. 2014;15:305-14.

16. Mauer AM, Kraut EH, Krauss SA, Ansari RH, Kasza K, Szeto $\mathrm{L}$, et al. Phase II trial of oxaliplatin, leucovorin and fluorouracil in patients with advanced carcinoma of the esophagus. Ann Oncol. 2005;16:1320-5.

17. Hsu PK, Huang CS, Wang BY, Wu YC, Hsu WH. Survival benefits of postoperative chemoradiation for lymph node-positive esophageal squamous cell carcinoma. Ann Thorac Surg. 2014;97:1734-41.

18. Lee J, Lee KE, Im YH, Kang WK, Park K, Kim K, et al. Adjuvant chemotherapy with 5-fluorouracil and cisplatin in lymph node-positive thoracic esophageal squamous cell carcinoma. Ann Thorac Surg. 2005;80:1170-5. 